\title{
,Differenzierte Integration': Konzept mit sprengender oder unitarisierender Kraft für die Europäische Union?
}

\author{
Peter-Christian Müller-Graff*
}

\begin{abstract}
,Differenzierte Integration`: Ein Konzept mit sprengender oder unitarisierender Kraft für die Europäische Union - eine Frage, die seit dem Anstieg der Mitgliedstaaten von 15 auf 27, den beiden gescheiterten Referenden zum Verfassungsvertrag, dem Dissens der Mitgliedstaaten in der Irakfrage und den allfälligen Erweiterungsdiskussionen latente Aktualität gewonnen hat. Sie umfasst die Fragenvarianten nach der, differenzierten Integration“ innerhalb und außerhalb des einheitlichen institutionellen Rahmens der Europäischen Union ebenso wie den Gedanken der Neugründung durch eine Avantgarde. Systemlozierte Antworten lassen sich dazu nur entwickeln, wenn zunächst begrifflich Farbe bekannt wird zur Redeweise von der , differenzierten Integration“ und ihren verschiedenen Dimensionen.
\end{abstract}

\section{Die verschiedenen Dimensionen der, differenzierten Integration“}

Der Topos der ,differenzierten Integration' kann nicht auf einen im Detail präzise konsentierten Sprachgebrauch zurückgreifen, sondern versteht sich nur allgemein als ein Abweichen von der homogenen Integration aller Mitgliedstaaten. Jenseits dessen registrierbar ist in der öffentlichen Diskussion, aber teilweise auch im wissenschaftlichen Schrifttum, jedoch ein nahezu babylonisches Kommunikationsdurcheinander ${ }^{1}$, das jeden Beitrag relativiert, der sein Verständnis nicht diszipliniert und vorweg erklärt. Nachfolgend sollen mit dem Terminus der , differenzierten Integration ‘ alle Formen des spezifischen, den gemeinsamen Acquis überschreitenden Zusammenwirkens einer Teilmenge von Mitgliedstaaten der

* Prof. Dr. Dr. h.c. Peter-Christian Müller-Graff, Universität Heidelberg.

Der Text beruht auf einem Vortrag, den der Verfasser auf Einladung der ECSA-Austria auf der Drei-LänderTagung der ECSA-Austria, des Arbeitskreises Europäische Integration und der ECSA-Schweiz zum Thema ,Differenzierte Integration“ in Wien im November 2005 gehalten hat.

1 Vgl. zur variierenden begrifflichen Vielfalt jüngst Wolfgang Wessels: Deutsche Europapolitik - Strategien für einen Wegweiser: Verstärkter Nutzen durch verbesserte Integration?, in: Wolfgang Wessels/Udo Diedrichs (Hrsg.): Die neue Europäische Union: im vitalen Interesse Deutschlands?, Studie der Europäischen Bewegung Deutschland, Berlin 2006, S. 155. Als frühe Thematisierungen aus rechtswissenschaftlicher Sicht vgl. Eberhard Grabitz/Constantin Iliopoulos: Typologie der Differenzierungen und Ausnahmen im Gemeinschaftsrecht, in: Eberhard Grabitz (Hrsg.): Abgestufte Integration: eine Alternative zum herkömmlichen Integrationskonzept? Ergebnisse eines Forschungsprojektes des Institutes für Integrationsforschung der Stiftung Europa-Kolleg Hamburg, Kehl am Rhein 1984, S. 31; Bernd Langeheine: Europäisches Gemeinschaftsrecht und abgestufte Integration, Berlin 1988. Speziell zur verstärkten Zusammenarbeit vgl. Veronika Grieser: Flexible Integration in der Europäischen Union: Neue Dynamik oder Gefährdung der Rechtseinheit?, Berlin 2003 (mit abgrenzender Begriffsbildung, S. 33); Ulrich Derpa: Die verstärkte Zusammenarbeit im Recht der Europäischen Union, Stuttgart 2003; vgl. zu Einzelfragen u.a. auch Astrid Epiney/Andreas Furrer: Umweltschutz nach Maastricht. Ein Europa der drei Geschwindigkeiten?, in: Europarecht 4/1992, S. 369-408, hier S. 369; Josef Janning: Europa braucht verschiedene Geschwindigkeiten, in: Europa-Archiv 18/1994, S. 527-536; John A. Usher: Variable Geometry or Concentric Circles: Patterns for the European Union, in: International and Comparative Law Quarterly 2/1997, S. 243-273; Nina Ost: Flexibilität des Gemeinschaftsrechts - Vom Notantrieb zum Vertragsprinzip?, in: Die Öffentliche Verwaltung 11/1997, S. 495; Hubert Isak: Der Gedanke der Rechtseinheit mit Blick auf den Beitritt mittel- und osteuropäischer Staaten, in: Europarecht, Beiheft 1/1998, 71ff.; Bernd Martenczuk: Die differenzierte Integration und die föderale Struktur der Europäischen Union, Europarecht 3/2000, S. 351-364; Christian von Buttlar: Rechtsprobleme der ,,verstärkten Zusammenarbeit“ nach dem Vertrag von Nizza, Zeitschrift für Europarechtliche Studien 4/2001, 649ff.; Claus Giering/Josef Janning, Flexibilität als Katalysator der Finalität? Die Gestaltungskraft der „Verstärkten Zusammenarbeit“ nach Nizza, in: integration 2/2001, S. 146-155. 
Europäischen Union erfasst werden, unabhängig davon, ob dies supranational oder intergouvernemental geschieht. Dieses Zusammenwirken ist sowohl modellförmig als auch inhaltlich aufgliederbar.

\section{Modellformen der , differenzierten Integration “}

Modellhaft lassen sich die Formen der ,differenzierten Integration" unter Aufnahme kurrenter geometrisch-physikalischer Sprachbilder rechtlich und politisch untergliedern in das Konzept der verschiedenen Geschwindigkeiten, das Konzept der konzentrischen Kreise, das Konzept der variablen Geometrie und den Gedanken des Europa à la carte.

Konzept der verschiedenen Geschwindigkeiten: Das Konzept der verschiedenen Geschwindigkeiten lässt sich in der Prämisse radizieren, dass zwar alle Mitgliedstaaten sich in den materiellen Einzelpolitiken und Einzelfragen auf dasselbe Ziel verpflichten, jedoch nur eine Teilmenge von Mitgliedstaaten dieses Ziel zu demselben Zeitpunkt verwirklichen kann oder will. Ausdruck dieses Konzepts sind namentlich Übergangsbestimmungen in den bisherigen Beitrittsverträgen, die die Erfüllung einer Pflicht (zum Beispiel Angleichungspflichten im Umweltrecht oder die Verwirklichung der Arbeitnehmerfreizügigkeit) terminlich hinausschieben. Zwar nicht geplant, aber de facto kann es zu unterschiedlichen Geschwindigkeiten auch im Falle der unterschiedlichen Befolgung von Harmonisierungspflichten binnenmarktintegrativer Richtlinien oder von anderen gemeinschaftsrechtlichen Pflichten kommen. Wegen dieses Dauerproblems mag man fragen, ob ,differenzierte Integration“ nicht eine allfällige Wirklichkeit der europäischen Integration darstellt. Das Modell der verschiedenen Geschwindigkeiten kennzeichnet auch den kriterienabhängigen Eintritt in die Währungsunion der zu diesem Währungsraum willigen Staaten ${ }^{2}$. Der Primärrechtsrahmen der Europäischen Gemeinschaft zur Währungsunion ist aber vielgestaltiger und leitet zugleich auch über zu dem Konzept der konzentrischen Kreise.

Konzept der konzentrischen Kreise: Das Konzept der konzentrischen Kreise lässt sich dadurch kennzeichnen, dass bereits nur eine Teilmenge von Mitgliedstaaten sich auf dieselben Ziele verpflichtet, wohingegen andere Mitgliedstaaten nicht mitziehen. Bei einem derartigen Vorgehen bildet sich eine Kerngruppe von mitwirkungswilligen Staaten, wohingegen die nicht teilnehmenden Mitgliedstaaten gewissermaßen einen schwächer ausgeprägten Kreis um die Kerngruppe bilden. Ausdruck dieses Konzepts war namentlich die Entstehung des Schengen-Raumes ${ }^{3}$ vor dessen Integration in den EG- und EU-Vertrag. Auch nach der Umgestaltung dieses Bereichs durch den Reformvertrag von Amsterdam 4 in den terminologisch so gefassten „Raum der Freiheit, der Sicherheit und des Rechts“5 bestehen noch Nachwirkungen des Konzepts der konzentrischen Kreise im Hinblick auf Dänemark, Großbritannien und Irland. ${ }^{6}$ Ausdruck des Konzepts der konzentrischen Kreise war auch das Sozialabkom-

2 Vgl. Art. 121 Abs. 3 EGV.

3 Dazu Hans Claudius Taschner: Schengen. Das Übereinkommen zum Abbau der Personenkontrollen an den Binnengrenzen von EU-Staaten, Baden-Baden 1997.

4 Vgl. dazu Protokoll zur Einbeziehung des Schengen-Besitzstands in den Rahmen der Europäischen Union; dazu Peter-Christian Müller-Graff: Institutionelle und materielle Reformen in der Zusammenarbeit in den Bereichen Justiz und Inneres, in: Waldemar Hummer (Hrsg.): Die Europäische Union nach dem Vertrag von Amsterdam, Wien 1998, S. 259-278; Peter-Christian Müller-Graff: Die fortentwickelnde Übernahme des Acquis der „Dritten Säule“ in die „Erste Säule“ der Union, in: Waldemar Hummer (Hrsg.): Rechtsfragen der Anwendung des Amsterdamer Vertrages, Wien 2001, S. 53-75.

5 Vgl. dazu Peter-Christian Müller-Graff: Der Raum der Freiheit, der Sicherheit und des Rechts. Der primärrechtliche Rahmen, in: Peter-Christian Müller-Graff (Hrsg.): Der Raum der Freiheit, der Sicherheit und des Rechts, Baden-Baden 2005, S. 11-27.

6 Ebenda. 
men von Maastricht zwischen elf der seinerzeit zwölf Mitgliedstaaten ${ }^{7}$, das dann allerdings gleichfalls durch den Reformvertrag von Amsterdam in den EG-Vertrag integriert wurde ${ }^{8}$. Prominentestes gegenwärtiges Beispiel ist aber die Währungsunion, zu deren Mitwirkung sich Großbritannien und Dänemark nicht ohne zusätzliche Erklärung verpflichtet haben ${ }^{9}$, wohingegen für Schweden eine derartige Sonderregelung nicht gilt und dessen Nichtteilnahme an der Währungsunion trotz Erfüllung der Konvergenzkriterien daher der Sache nach den Vertragsverpflichtungen widerspricht. ${ }^{10}$

Konzept der variablen Geometrie: Als Konzept der variablen Geometrie lässt sich ein Ordnungsmodell bezeichnen, das eine identische Teilmenge von Mitgliedstaaten in verschiedenen Bereichen des Zusammenwirkens nicht voraussetzt. In diesem Fall bildet sich keine randscharfe Kerngruppe. Vielmehr kann die Zusammensetzung der Mitwirkenden in den einzelnen einer Differenzierung unterliegenden Sachfragen variieren. Im Ansatz wird davon das Gesamtbild des Status quo der Differenzierungen gekennzeichnet, dies allerdings vor dem Hintergrund eines grundsätzlichen Homogenitätsprinzipes im Sinne des Grundsatzes, dass alle Mitgliedstaaten in gleicher Weise teilnehmen und zur selben Zeit dieselben Inhalte verwirklichen. Ungeachtet dieses Grundsatzes existieren gegenwärtig aber eben auch Elemente der in diesem Sinne differenzierten Integration - und zwar ohne Nutzung des EG- und EU-vertraglichen Instruments der verstärkten Zusammenarbeit der Art. 43-45 EUV und Art.11 EGV. Die Zusammensetzung in den verwirklichten Differenzierungen variiert. So unterscheidet sich die Staatenkonfiguration namentlich beim Zusammenwirken des „Raumes der Freiheit, der Sicherheit und des Rechtes"11 von derjenigen in der Währungsunion ${ }^{12}$ und diese wiederum von der vollen Teilnahme am Binnenmarkt im Verhältnis der Altmitgliedstaaten und den meisten neuen Beitrittsstaaten des Jahres 2004 und 2007. 13

Der Gedanke eines ,Europa à la carte': Der Gedanke eines Europa à la carte ist dem Konzept der variablen Geometrie in seiner Wirkung verwandt. Denn auch hier kommt es nicht zu einer randscharfen Kerngruppe. Allerdings wird mit ,Europa à la carte“ nicht ein Zustand oder ein Modell bezeichnet, sondern lediglich ein Auswahlvorgang. Er kommt überdies ohne die Gemeinschaft als begrenzenden Faktor für die Teilnahme aus, wie es das Europäische Patentübereinkommen in seinen Anfängen illustrierte ${ }^{14}$ - im Unterschied zu

7 Otto Schulz: Maastricht und die Grundlagen einer Europäischen Sozialpolitik, Köln 1996, S. 61.

8 Vgl. Robert Rebhahn in: Jürgen Schwarze (Hrsg.): EU-Kommentar, Baden-Baden 2000, Art.136 EGV Rn.4.

9 Vgl. Protokoll (Nr. 25) über einige Bestimmungen betreffend das Vereinigte Königreich Großbritannien und Nordirland; Protokoll (Nr. 26) über einige Bestimmungen betreffend Dänemark.

10 Vgl. zur schwedischen Erfüllung der Konvergenzkriterien Gegensätze im hohen Norden, in: Frankfurter Allgemeine Zeitung, 03.01.2006, S.12; zur Teilnahmepflicht bei Erfüllung der Voraussetzungen Ulrich Häde: Zur Rechtmäßigkeit der Entscheidung über die Europäische Wirtschafts- und Währungsunion, in: Juristenzeitung 22/1998, S. 1088-1092.

11 Schulz: Maastricht und die Grundlagen einer Europäischen Sozialpolitik.

12 Vgl. Protokoll (Nr.25) über einige Bestimmungen betreffend das Vereinigte Königreich Großbritannien und Nordirland; Protokoll (Nr.26) über einige Bestimmungen betreffend Dänemark. Seit 01.01.2007 haben 13 von 27 Staaten den Euro als gemeinsame Währung.

13 Vgl. dazu den räumlichen Anwendungsbereich der konstituierenden Normen des Binnenmarktrechts im einzelnen im Vergleich von Sonderbestimmungen in Beitrittsverträgen, Protokollen für Altmitgliedstaaten (z.B. Protokoll über die Anwendung bestimmter Aspekte des Artikels 14 EGV auf das Vereinigte Königreich und auf Irland) und von Übergangs- und Sonderbestimmungen in der Beitrittsakte zur Erweiterung des Jahres 2004 und 2007.

14 Übereinkommen über das europäische Patent für den Gemeinsamen Markt vom 05.10.1973; dazu Peter-Christian Müller-Graff: Europäisches Patent, in: Joerg Monar/Nanette Neuwahl/Paul Noack (Hrsg.): Sachwörterbuch zur Europäischen Union, Stuttgart 1993, S.128; Romuald Singer/Margarete Singer: Europäisches Patentübereinkommen, Köln 1989; Münchener Gemeinschaftskommentar: Europäisches Patentübereinkommen, 1991. 
dem niemals in Kraft getretenen Gemeinschaftspatentübereinkommen ${ }^{15}$, das von allen Mitgliedstaaten, aber nur von diesen unterzeichnet war.

\section{Erfasste Gegenstände}

Die Ausweisung des Gedankens der , differenzierten Integration' in verschiedenen Modellformen beantwortet naturgemäß noch nicht die Frage, auf welchen Gegenstand sie sich sinnfällig beziehen können. ,Differenzierte Integration“ ist zunächst nur ein rechtlich und politisch blutleeres abstraktes Wort. Vertieft man indes diesen Topos, so ist, differenzierte Integration' grundsätzlich sowohl sachgebietlich als auch organisationell oder institutionell denkbar.

Sachgebietliche Differenzierung: Sachgebietlich wird man zunächst zu fragen haben, ob , differenzierte Integration “ im Sinne zusätzlicher Vertiefung nicht möglicherweise eine Chimäre steriler politischer oder akademischer Rhetorik ist. So ist denkbar, dass möglicherweise bereits jetzt die ernsthaft und realitätsfähig denkbare inhaltliche Finalität der europäischen Verbandskompetenz im Wesentlichen erreicht ist. Diese Frage stellt sich insbesondere, wenn man bedenkt, dass der größte Mitgliedstaat von Verfassungs wegen nur an einer Europäischen Union mitwirken kann, die föderativen Grundsätzen verpflichtet ist (Art. 23 Abs. 1 GG). Dies beinhaltet eine Achtung der substanziellen Autonomie ihrer Mitglieder. ${ }^{16}$ Freilich bedeutet andererseits die Begründung einer Verbandskompetenz für die europäische Ebene nur die Existenz einer abstrakten Handlungsbefugnis, noch nicht aber auch deren tatsächliche Ausschöpfung. Dies zeigt sich umso deutlicher, wenn zu deren Ausübung Einstimmigkeit im Rat erforderlich ist. ${ }^{17}$

Sachgebietlich lässt sich in diesem Sinne ein verstärktes Zusammenwirken einer Teilmenge von Mitgliedstaaten vor dem Hintergrund des erreichten Integrationsstands daher beispielsweise insbesondere in folgenden Bereichen vorstellen: in Bereichen der polizeilichen und institutionellen Zusammenarbeit in Strafsachen einschließlich des Strafverfahrensrechtes und des materiellen Strafrechts ${ }^{18}$, des Weiteren in der Einwanderungs- und Asylpolitik $^{19}$, ferner in der ziviljustiziellen Zusammenarbeit im Zivilrecht ${ }^{20}$, in der Wirtschafts-, Sozial- und Steuerpolitik ${ }^{21}$ und schließlich auch in der allgemeinen Außen-, Sicherheits- und Verteidigungspolitik. ${ }^{22}$ Allerdings ergibt sich, wie schon angesprochen, ein verstärktes $\mathrm{Zu}$ sammenwirken auch in allen bereits bestehenden Befugnisbereichen der Gemeinschaft oder Kooperationsbereichen der Union, in denen nicht alle 27 Mitglieder das Zusammenwirken faktisch verwirklichen.

Organisationelle und institutionelle Differenzierung: In organisationeller und institutioneller Hinsicht kann das verstärkte Zusammenwirken einer Teilmenge von Mitgliedstaaten vor allem die Ausgestaltung der transnationalen organisationellen und institutionellen Verflechtung zwischen ihnen beinhalten. Dies kann bis hin zum Zusammenschluss einiger Mitgliedstaaten zu einem Staat gedacht werden: so beispielsweise hypothetisch im Zusammenschluss der Benelux-Staaten zu einem Bundesstaat. Eine derartige föderale Variante des

15 Übereinkommen über das europäische Patent für den Gemeinsamen Markt vom 15.2.1975/15.12.1989; dazu Müller-Graff: Europäisches Patent, S. 184-185.

16 Vgl. auch die verfassungsrechtlichen Anforderungen nach BVerfG, EuGRZ 1993, 429ff.

17 Zum Beispiel Artt.93, 94 EGV.

18 Zum primärrechtlichen Unionsacquis vgl. Art. 29ff. EUV.

19 Zum primärrechtlichen Gemeinschaftsacquis vgl. Art. 61ff. EGV.

20 Zum primärrechtlichen Gemeinschaftsacquis vgl. Art.65 EGV.

21 Zum primärrechtlichen Gemeinschaftsacquis vgl. Art. 93, 98, 136ff. EGV.

22 Zum primärrechtlichen Unionsacquis vgl. Art.11ff. EUV. 
Zusammenwirkens wurde vor einigen Jahren in der politischen Diskussion um die europäischen Zukunftsperspektiven vereinzelt speziell für das Verhältnis zwischen Deutschland und Frankreich artikuliert. Keine Differenzierung, sondern das Ende der vertraglich verbindlichen Integrationsbeteiligung ist demgegenüber der Austritt eines Mitglieds oder ein Austrittsvertrag (dessen Abschluss auch der Angebotsinitiative der anderen Mitglieder entspringen kann, wenn ein Mitglied dauerhaft schwerwiegend wesentliche Mitgliedschaftspflichten verletzt).

\section{Einzelthesen zur Frage ,Sprengsatz oder unitarische Kraft?‘}

Vor diesem Verständnishintergrund lassen sich zu der Frage, ob die ,differenzierte Integration" einen Sprengsatz darstellt oder aber unitarische Kraft entfalten kann, fünf aufeinander aufbauende Thesen entwickeln.

\section{Die Notwendigkeit der Unterscheidung des Zeithorizonts}

Bei Konzepten der ,differenzierten Integration“ ist im Hinblick auf deren sprengende oder unitarisierende Wirkung zuallererst nach dem Zeithorizont zu unterscheiden.

Die kurzfristige Desintegrationswirkung: Es liegt auf der Hand, dass jede Art differenzierter Integration konzeptionell und faktisch jedenfalls kurzfristig eine Abweichung vom Homogenitätsgrundsatz der Mitgliedschaft beinhaltet und daher bei kurzfristiger Betrachtung weder unitarisierend angelegt ist noch wirkt. Im Gegenteil soll unterschiedlichen Fähigkeiten oder unterschiedlicher Bereitschaft zur Verwirklichung eines gemeinsamen Standards Rechnung getragen werden. So gesehen begünstigen Konzepte differenzierter Integration grundsätzlich einen Rückfall in die Beliebigkeit und Heterogenität klassischer Bündnispolitik. Denn je weniger Druck zur Gemeinsamkeit besteht, desto eher entstehen Bündnisse zu Einzelthemen.

Die mittel- und langfristige Perspektive: Ob sich hinter der kurzfristigen Desintegrationswirkung eine listige Strategie oder ein selbstwirksamer Mechanismus verbirgt, alle Mitgliedstaaten im betroffenen Bereich mittel- oder langfristig unitarisierend mitzuziehen, lässt sich nicht abstrakt, sondern nur für den jeweiligen einzelnen Differenzierungsschritt erörtern und analysieren.

Unitarisierungsbeispiele: Aus der vermeintlich einfachen Retroperspektive betrachtet (vermeintlich einfach, weil allzu leicht Koinzidenz mit Kausalität verwechselt werden kann), haben einige der wichtigsten Differenzierungsfälle mittelfristig nicht gespalten, sondern tendenziell unitarisiert. Dies gilt für das Sozialabkommen von Maastricht ${ }^{23}$, für die Schengen-Abkommen ${ }^{24}$ (allerdings hier schon mit Ausnahmen ${ }^{25}$ ), für alle bisherigen Beitritte im Hinblick auf Übergangsklauseln (mit Ausnahme der noch laufenden Übergangszeiten für die Beitrittsstaaten der Jahre 2004 und 2007) und in Großdimension sogar für die Gründung der EWG durch eine kleine Teilgruppe europäischer Kontinentalstaaten im Jahre 1957, die sich bis heute um 21 weitere Mitglieder erweitert hat.

Spaltungsbeispiele: Gleiches lässt sich für die Währungsunion bislang nicht sagen. Zu den am 1. Januar 1999 die Währungsunion begründenden Staaten ist bislang nur ein einziges weiteres Mitglied hinzugekommen (Slowenien). Im Gegenteil sind Anläufe in Schweden und Dänemark gescheitert und Großbritannien hat es nicht einmal versucht. ${ }^{26}$ Elf der zwölf Beitrittsstaaten der Jahre 2004 und 2007 sind aus unterschiedlichen Gründen noch nicht bei-

23 Schulz: Maastricht und die Grundlagen einer Europäischen Sozialpolitik.

24 Taschner: Schengen. Das Übereinkommen zum Abbau der Personenkontrollen.

25 Taschner: Schengen. Das Übereinkommen zum Abbau der Personenkontrollen.

26 Vgl. Gegensätze im hohen Norden, in: Frankfurter Allgemeine Zeitung, 03.01.2006, S.12. 
trittsreif. ${ }^{27}$ Im Schengen-Bereich haben sich drei Altmitgliedstaaten bis heute einer vollen Einbeziehung entzogen ${ }^{28}$ und es bleibt zu sehen, zu welchem Zeitpunkt alle Beitrittsstaaten der Jahre 2004 und 2007 voll einbezogen sein werden.

Folgefrage: Dieser gemischte Erfahrungsbefund mit sachgebietlich ,differenzierter Integration" wirft die Folgefrage auf, ob sich daraus allgemeine Aussagen oder sogar Entwicklungsgesetzmäßigkeiten ableiten lassen. Dies führt zu einer zweiten These.

\section{Der Zusammenhang von Binnenmarktnähe und Unitarisierungswirkung}

Die zweite These lautet, dass eine mittelfristig integrativ unitarisierende Tendenz eines Differenzierungsschritts umso wahrscheinlicher ist, je stärker sie dem Funktionieren des Binnenmarktes dient und je weniger sie ein Widerlager spezifisch nationaler Politikinteressen findet. Anders gesagt: Die vom Prinzip des komparativen Kostenvorteils ${ }^{29}$ (Art. 14 Abs. 2 EGV) entfesselten Vernetzungskräfte des gemeinsamen europäischen Wirtschaftsraums generieren Bedürfnisse und Zusammenwirken in binnenmarktnahen Bereichen. Stoßen die Vernetzungskräfte auf Widerstände von Politikinteressen in einer Teilmenge anderer Mitgliedstaaten, können sie erstens den Ausweg einer, differenzierten Integration' suchen und bewirken zweitens die Tendenz, mittel- oder langfristig die ursprünglich fern stehenden Staaten miteinzubeziehen.

Unterscheidet man nach dem Nähegrad zur Binnenmarktverwirklichung, lassen sich im Licht der bisherigen Fälle drei Gruppen bilden: die binnenmarktzwingenden Fälle, die binnenmarktplausiblen Fälle und die binnenmarktfernen Fälle.

\section{Die binnenmarktzwingenden Fälle}

Bei der ersten Gruppe handelt es sich um die binnenmarktfunktional erforderlichen, also binnenmarktzwingenden Folgeschritte.

Binnenmarktliche Personenfreizügigkeit: Eine besonders deutliche funktionale Binnenmarktnähe weisen ganz offensichtlich Fragen auf, die wegen des Ziels der binnenmarktlichen Personenfreizügigkeit ${ }^{30}$ aus dem konzipierten oder verwirklichten Wegfall von Personenkontrollen an den innergemeinschaftlichen Grenzen entstehen. Dieser Schritt entsprach dem durch die Einheitliche Europäische Akte im Jahre 1987 in das Primärrecht eingefügten Ziel, einen Raum ohne Binnengrenzen zu schaffen, in dem der freie Verkehr von Waren, Personen, Dienstleistungen und Kapital gewährleistet ist. ${ }^{31}$ Konsequenterweise enthielt damit die Frage Gewicht, ob der binnenmarktlich angestrebte Wegfall der (vom begleitenden Weißbuch der Kommission seinerzeit sogenannten) physischen Grenzen durch die Bedürfnisse nach Grenzkontrollen zum Schutz gegen unerwünschten Zuzug von Drittstaatern und zum Schutz gegen die Freizügigkeit von Straftätern und organisierter Kriminalität blockiert würde. ${ }^{32}$ Folgerichtig erkannte man die Notwendigkeit, den Wegfall der Personenkontrollen zu kompensieren und konzipierte die Kompensation in einer Koordinierung der nationalen Zugangspolitiken (Visa-, Einwanderungs- und Asylpolitik) ebenso wie in einer Koordinie-

27 Vgl. dazu die Bilanz: Fast allen Kandidaten fehlt die Euro-Reife, in: Frankfurter Allgemeine Zeitung, 03.01.2006, S. 12 .

28 Vgl. Müller-Graff: Der Raum der Freiheit, der Sicherheit und des Rechts, S. 11-27.

29 Zu diesem Konzept z.B. Willem Molle: The Economics of European Integration, Aldershot 1991, S. 9.

30 Art. 14 Abs. 2, Art. 39 ff., Art. 49 EGV.

31 Vgl. dazu Peter-Christian Müller-Graff: Die Rechtsangleichung zur Verwirklichung des Binnenmarktes, in: Europarecht 2/1989, S. 107-151.

32 Zur letztgenannten Frage vgl. Hans-Heiner Kühne: Die Bekämpfung von internationaler Kriminalität und von Drogenabhängigkeit als gemeinsames Interesse der Unionsstaaten, in: Müller-Graff (Hrsg.): Europäische Zusammenarbeit in den Bereichen Justiz und Inneres, S. 85-99. 
rung der grenzüberschreitenden Kriminalitätsbekämpfung. Zunächst fand sich die mitgliedstaatliche Teilmenge der Schengen-Staaten mit zwei Abkommen zu einem verdichteten $\mathrm{Zu}$ sammenwirken in diesen Aufgaben zusammen. ${ }^{33}$ Für alle Mitgliedstaaten brachte den Kompensationsgedanken sodann Artikel K des Maastrichter Unionsvertrages folgerichtig darin zum Ausdruck, dass er diese Bereiche zu Gegenständen von gemeinsamem Interesse der Mitgliedstaaten erhob ${ }^{34}$. Lediglich fünf Jahre nach Maastricht erfolgte, wie bereits aufgewiesen $^{35}$, im Amsterdamer Reformvertrag, ungeachtet der genannten Ausnahmen für drei Mitgliedstaaten, eine konzeptionell sinnfällige Übernahme des Schengen-Bestandes in den Rahmen von EG und EU-Vertrag. Zugleich wurde vor allem auch eine konzeptionell und systematisch konsequente Lokation der genannten Zugangspolitiken in den Art. 61-69 EGV im unmittelbaren Anschluss an die Marktfreiheiten des Binnenmarktes, nämlich nach dem Abschnitt über die Personen-, Dienstleistungs- und Kapitalverkehrsfreiheit, vorgenommen. Überdies sieht der Verfassungsvertrag für die Reste der dritten Säule eine europäische Verbandskompetenz vor. ${ }^{36}$ Unabhängig davon könnte das Abkommen von Prüm eine ähnliche Wirkung wie das seinerzeitige Schengen-Abkommen entfalten. ${ }^{37}$

Sozialpolitik: Einen zweiten Bereich von großer Binnenmarktnähe bildet die Sozialpolitik. In ihrem Ursprung von Anbeginn an als Teil des EWG-Vertrages zur Dämpfung von in Sozialkosten begründeten Wettbewerbsverzerrungen im Gemeinsamen Markt und zur Förderung der binnenmarktlichen Arbeitnehmerfreizügigkeit konzipiert ${ }^{38}$, beinhaltete das Sozialabkommen von Maastricht eine marktintegrativ funktional sinnfällige und potenziell über die Marktintegration hinausgehende Weiterentwicklung. Elf von zwölf Mitgliedstaaten wurde durch ein Protokoll zum Vertrag von Maastricht gestattet, in der Sozialpolitik innerhalb der Institutionen und Verfahren der Gemeinschaft intensiver zusammenzuwirken ${ }^{39}$. Großbritannien, seinerzeit mit konservativer Regierung, blieb abseits. Die nachfolgende Labour-Regierung vollzog in Amsterdam den sozialpolitischen Schulterschluss, sodass auch insoweit eine letztlich aus der binnenmarktlichen System- und Folgelogik generierte Unitarisierung bewirkt wurde.

Die Außenwirkung des Binnenmarktkonzepts: Die Sogkraft des Binnenmarktes zeigt sich überdies vor allem darin, dass seine unitarisierende Kraft weit über die Mitgliedstaaten hinausreicht und insoweit das binnenmarktliche Zusammenwirken aller Mitgliedstaaten als Form einer, differenzierten Integration` im Gesamtkreis aller europäischen Staaten erkennen lässt. Abgesehen von der allmählichen Anziehungskraft auf EFTA-Mitglieder unter Aushöhlung des Mitgliederbestandes der EFTA, beginnend schon mit den ersten Absetzungserscheinungen Großbritanniens von seiner eigenen Initiative im Jahre 1961, und abgesehen auch von den konsekutiven Beitritten seit 1973, finden sich insoweit markante Auswirkungen auf das Recht von drei der vier verbliebenen EFTA-Staaten im Rahmen des EWR-Abkommens. ${ }^{40}$ Aber auch die Schweiz entzieht sich nicht diesem unitarisierenden Sog der in

33 Vgl. Müller-Graff: Der Raum der Freiheit, der Sicherheit und des Rechts, S. 11-27.

34 Ebenda.

35 Ebenda.

36 Art. III-270ff. VVE.

37 Vgl. Daniela Kietz/Andreas Maurer: Der Vertrag von Prüm: Vertiefungs- und Fragmentierungstendenzen in der Justiz- und Innenpolitik, in: integration 3/2006, S. 201-212.

38 Rolf Birk: Das Arbeitsrecht der Europäischen Gemeinschaft, in: Reinhard Richardi/Manfred Wlotzke (Hrsg.): Münchener Handbuch zum Arbeitsrecht, Band 1, München 1992, § 18 Rn. 5ff.; Rebahn: Art.136 EGV Rn. 5, der allerdings in der Neufassung durch Art. 136 EGV eine Lösung der Sozialpolitik vom „engen Verbund mit der Wettbewerbspolitik“ und ,erstmals eine genuin sozialpolitische Kompetenz der EG zur Rechtsetzung“" erkennt.

39 Vgl. Rebhahn: Art. 136 EGV Rn. 4.

40 Vgl. zu diesem namentlich Olivier Jacot-Guillarmod (Hrsg.): Accord EEE/EWR-Abkommen, Zürich/Bern 1992; Peter-Christian Müller-Graff/Erling Selvig (Hrsg.): The European Economic Area, Berlin/Oslo 1997; Carl Baudenbacher/Per Tresselt/Thorgeir Örlygsson (Hrsg.): The EFTA Court - Ten Years on, Oxford/Portland 2005. 
der EG zusammengeschlossenen Teilmenge europäischer Staaten. ${ }^{41}$ Der jüngste Aufwuchs bilateraler Abkommen ${ }^{42}$ und der sogenannte autonome Nachvollzug ${ }^{43}$ mit dem damit verbundenen materiellen Souveränitätsverlust legen Zeugnis davon ab. Die Europa-Abkommen mit zehn mittel- und osteuropäischen Staaten ${ }^{44}$, die durch deren Beitritt in den Jahren 2004 und 2007 hinfällig geworden sind, runden dieses Bild der unitarisierenden Kraft des Konzepts der Marktintegration durch die Initiative einer ursprünglichen kleinen Gruppe von Staaten ab.

\section{Die binnenmarktplausiblen Fälle}

Eine zweite, wiewohl weniger zwingende Binnenmarktnähe weisen diejenigen Fälle auf, die zum Funktionieren des Binnenmarktes zwar nicht notwendig, jedoch sehr plausibel sind.

Währungsunion: Die Binnenmarktplausibilität betrifft vor allem die Währungsunion. $\mathrm{Ob}$ diese für das Funktionieren des Binnenmarktes notwendig ist, ist zwar letztlich eine politische Frage der Intensitätsdefinition des gewünschten Binnenmarktes ${ }^{45}$. Dennoch ist erkennbar, dass eine Währungsunion höchst sinnfällig ist, um im binnenmarktlichen Wirtschaftsraum das Problem breitflächiger Wettbewerbsverzerrungen infolge der Wechselkursschwankungen zwischen verschiedenen einzelstaatlichen Währungen und damit eine latente Beeinträchtigung der Funktionsfähigkeit der Grundfreiheiten zu lösen. Die , differenzierte Integration ‘ der Währungsunion entfaltet damit grundsätzlich einen Anreiz für die anderen, am binnenmarktlichen Austausch teilnehmenden Mitgliedstaaten, derartige Verwerfungen auch durch Teilnahme an der gemeinsamen Währung zu bewältigen. Da allerdings mit der Währungshoheit ein zentraler Teil von Staatlichkeit betroffen ist ${ }^{46}$, bestehen insoweit gegenläufige Eigenkräfte. ${ }^{47}$ Sie enthalten auf Dauer ein Distanzierungspotenzial und schmälern das politische Gewicht nicht teilnehmender Staaten innerhalb der Europäischen Union schon mangels vergleichbarer Betroffenheit und infolge des Gruppeneffekts und Korpsgefühls der an der Währunsunion teilnehmenden Staaten. Eine ebenbürtige Mitführungsrolle in der Union zu beanspruchen, ohne Mitglied der Währungsunion zu sein, erschiene daher zweifelhaft.

Strafverfolgung und Polizeitätigkeit: Ein zweiter Fall der relativen, aber nicht zwingenden Binnenmarktnähe, ist die Koordinierung der Strafverfolgung und Polizeitätigkeit. Die Grenzlinien des funktional zwingenden Zusammenwirkens zu der lediglich förderlichen Unitarisierung sind in diesem Bereich unklar. So ist durchaus zweifelhaft, ob es binnenmarktlich, aber auch allgemein des Grundsatzes der gegenseitigen Anerkennung aller gerichtlichen und außergerichtlichen Entscheidungen im Rahmen der justiziellen Zusammenarbeit in Strafsachen bedarf, wie es der Verfassungsvertrag vorsieht. ${ }^{48}$

41 Vgl. dazu namentlich die Beiträge in: Peter Forstmoser/Hans Caspar von der Crone/Rolf H. Weber/Dieter Zobl (Hrsg.): Der Einfluß des europäischen Rechts auf die Schweiz. Festschrift für Roger Zäch, Zürich 1999.

42 Astrid Epiney: Zu den ,bilateralen“ Verträgen der EG bzw. ihren Mitgliedstaaten und der Schweiz, in: Zeitschrift für Gemeinschaftsprivatrecht 1/2006, S. 1-52, hier S. 2.

43 Vgl. z.B. Wolfgang Wiegand: Zur Anwendung von autonom nachvollzogenem EU-Privatrecht, in: Peter Forstmoser u.a. (Hrsg.): Der Einfluß des europäischen Rechts auf die Schweiz, S.171; Heinrich Koller: Autonomer Nachvollzug - Editorial, in: Zeitschrift für Gemeinschaftsprivatrecht 1/2006.

44 Vgl. Peter-Christian Müller-Graff: Die rechtliche Dimension der Osterweiterung der Europäischen Union, in: Wolfgang Heusel (Hrsg.): Die Osterweiterung der Europäischen Union, Köln 2002, S. 21-48.

45 Vgl. Jean-Francois Pons: L’Union Economique et Monétaire et la monnaie unique: le point de vue de la Commission, in: Manfred Willms (Hrsg.): European Monetary Union: Legal Foundations and Economic Implications, Luxemburg 1993, S.201ff.; auch Roland Vaubel: The European Monetary Union: a public choice approach, in: Willms (Hrsg.): European Monetary Union, S. 163ff.

46 Vgl. Charles Goodhart: The british position on EMU, in: Willms (Hrsg.): European Monetary Union, S. $217 \mathrm{ff}$.

47 Ebenda.

48 Vgl. namentlich Art. III-270 VVE. 


\section{Die binnenmarktfernen Fälle}

Eine dritte Kategorie von Sachpolitiken sind im Verhältnis zum Binnenmarktziel diejenigen Bereiche, die als binnenmarktfern bezeichnet werden können.

Allgemeine Außen- und Sicherheitspolitik: Zu dieser Gruppe zählt anders als die binnenmarktabsichernde Handelspolitik die allgemeine Außen- und Sicherheitspolitik. Zweifelsohne sichert gemeinsames Auftreten in diesem Bereich auch die Freude der Vertragspartner aneinander im Zusammenwirken im gemeinsamen Europäischen Wirtschaftsraum. Aber die Dissonanzen in der Irakfrage und die beiden dabei beobachtbaren konkurrierenden differenzierenden ,Integrationsbündnisse', sofern die Konvergenz einzelner nationaler Standpunkte überhaupt als ,Bündnis'bildung angesehen werden $\mathrm{kann}^{49}$, haben das Funktionieren des Binnenmarktes nicht erschüttert. Es wird eine vertiefungswürdige Frage sein, ob die Divergenzen als Schockerlebnis eine unitarisierende Tendenz in der außenpolitischen Problembehandlung künftig fördern. Bislang überwiegt eher der Eindruck, dass die Spaltung in der Irakfrage nachwirkende Sprengkraft enthält. Bis heute nicht grundsätzlich überbrückt scheint die Kluft in dieser Frage zwischen einer Reihe von Kontinentalstaaten zu Großbritannien und anderen Mitgliedstaaten, die Truppen im Irak unterhalten. Die Union wird sich nicht viele solcher Dissonanzen und ,differenzierter Integrationen' leisten können, soll ihr Funktionieren, ihr Zusammenhalt oder ihre Mitgliederkonfiguration nicht ernsthaft Schaden nehmen.

Institutionell-organisatorisches Zusammenwirken: Binnenmarktfern ist auch eine institutionell-organisatorische Verdichtung des Zusammenwirkens einer Teilmenge von Mitgliedstaaten. Diese spielt sich ohne darüber hinausgehende, institutionell fassbare unitarisierende oder spaltende Wirkungen ab, wie die Beispiele Benelux, Deutsch-Französische Zusammenarbeit oder Weimarer Dreieck zeigen. Selbst eine Föderation zwischen Deutschland und Frankreich müsste deshalb das Funktionieren des Binnenmarktes nicht notwenig erschüttern, würde aber auch keinen binnenmarktgetriebenen Sog zu einer breitflächigen europäischen Föderation entfalten.

\section{Die grundsätzliche Unerheblichkeit der inneren oder äußeren Differenzierung}

Die dritte These lautet, dass das integrationsfördernde oder integrationszerstörende Potenzial sachgebietlich differenzierter Integration grundsätzlich unabhängig davon ist, ob sie innerhalb oder außerhalb der Institutionen erfolgt, doch ist dies nur ein Grundsatz, der rasch Grenzen findet.

Diese Aussage lässt sich auf die Beobachtung des Schicksals der Schengen-Zusammenarbeit (begonnen außerhalb der EWG) und des Schicksals des Sozialabkommens (begonnen innerhalb der EG) stützen. In beiden Fällen kam es schließlich zu einer Homogenisierung innerhalb der EG beziehungsweise EU.

Allerdings mag man die fortdauernde Resistenz von Großbritannien, Dänemark und Irland gegenüber Teilen des Raums der Freiheit, der Sicherheit und des Rechts als Indiz dafür sehen, dass ein außerhalb der EU-Institutionen begonnenes Zusammenwirken auf stärkeren Widerstand gegen eine sachgegenständliche Unitarisierung stoßen kann. Dies hat gewiss

49 Ein derartiger Terminus darf nicht zu viel an Konsistenz innerhalb der beiden Gruppierungen signalisieren und beinhaltet hinsichtlich des ,Briefes der Acht" auch lediglich Gemeinsamkeit in der abstrakten grundsätzlichen Position, nicht aber in den konzeptionellen und operativen Folgerungen. Es mag in der Irak-Krise eher eine Nationalisierung der Standpunkte gesehen werden können als eine Bündnisbildung; vgl. dazu als substantiierte Analyse Bernhard Stahl: Understanding the "great split" in the Iraq crisis. A comparative approach of the member states' foreign policy, UACES Konferenz in Zagreb, 4.-7.09.2005, abrufbar unter: http://www.politik.uni-trier.de/forschung/Paper_Iraq.pdf (letzter Zugriff: 17.02.2007). 
verschiedene sachpolitisch erklärliche Ursachen. Einen der Gründe wird man aber wohl auch darin sehen müssen, dass in diesen Fällen der Anfangssegen aller Mitgliedstaaten für eine neue Gemeinsamkeit innerhalb des Rahmens der Europäischen Union fehlt. Dementsprechend ungebunden sind die Willigen in der Gestaltung ihres Differenzierungsschritts. Beim Sozialabkommen war das Einverständnis von Anfang an vorhanden. Daher lassen sich gegen die Ausweitung der Währungsunion jedenfalls keine Schwierigkeiten aus dem Gesichtspunkt mangelnder Anfangsabstimmung erwarten.

Freilich kann man daher bei aller wissenschaftlich gebotenen Zurückhaltung gegenüber Voraussagen auch folgern, dass die (wohl auch unvermeidlich affrontativ wirkende) ,Neugründung ' der Union durch eine (euphorisch dynamisch sogenannte), Avantgarde' (oder „tête du peloton“"50), also etwa die Gründung einer verfassungsvertraglich gegründeten Teilgruppe, nicht ohne Schwierigkeiten oder kaum damit rechnen könnte, eines Tages unitarisierend alle bisherigen Mitgliedstaaten an Bord zu hieven. Dies gilt umso mehr, als eine Verschlankung des Mitgliederkreises durch Neugründung gerade am Beginn eines derartigen Aktes stünde und wohl sogar deren Geburtsgrund wäre. Andererseits läßt sich aber auch nicht ausschließen, dass die ernsthafte Invokation eines derartigen Donnerschlages, wenn er überhaupt realistisch verfügbar ist, den Zusammenhalt zu fördern vermag.

\section{Differenzierung als Preis des Mitgliederzuwachses}

Die vierte These lautet, dass die Bedeutung ,differenzierter Integration' als Kompositionssystem der Union mit der Zunahme ihrer Mitglieder steigt. Integrationshistorisch auffällig entstand das Thema der , abgestuften Integration “ oder ,zwei Geschwindigkeiten ' bereits nach der ersten Erweiterung. ${ }^{51}$ Mit der EWG der Zwölf war dann der Punkt erreicht, an dem derartige Vorüberlegungen politische Realität wurden: nämlich in Gestalt der Schengen-Abkommen ${ }^{52}$, der Währungsunion ${ }^{53}$ und des Sozialabkommens. ${ }^{54}$ Noch ist nicht ausgemacht, ob die große Erweiterung der Jahre 2004 und 2007 nicht dauerhafte Unterschiede in der faktischen Integrationsdichte unter dem Gesichtspunkt der tatsächlichen Umsetzung von Gemeinschaftsrecht zur Folge haben wird. Der letzte umfassende Forschrittsbericht der Kommission vor dem Großbeitritt bescheinigte keineswegs eine rundum zufrieden stellende Verwirklichung des Acquis in allen Aktionsbereichen ${ }^{55}$. Als Querschnittsprobleme für nahezu alle seinerzeitigen mittel- und osteuropäischen Kandidatenstaaten erwiesen sich der Umweltschutz, die Beihilfenaufsicht und die justiziellen und adminstrativen Kapazitäten. Zur Kompensation für dieses Problem partiell mangelnder Beitrittsreife wurden deshalb neuartige Schutzklauseln für den Beitrittsvertrag geschaffen, die von Marek Zila plastisch

50 So Jacques Chirac: Rede des französischen Staatspräsidenten vor dem Deutschen Bundestag am 27.06.2000, abrufbar unter: http://www.bundestag.de/geschichte/gastredner/chirac/chirac1.html (letzter Zugriff: 28.02.2007).

51 Vgl. Willy Brandt: Rede des Vorsitzenden der Sozialdemokratischen Partei Deutschlands vor der Organisation Francaise du Mouvement Européen in Paris am 19.11.1974, in: Europa-Archiv 2/1975, S. D 33ff.; Leo Tindemans: Die Europäische Union, Bericht an den Europäischen Rat, EG-Bulletin, Beilage 1/1976; zur Entwicklung des Gedankens der abgestuften Integration Veronika Grieser: Flexible Integration in der Europäischen Union, S. 32.

52 Vgl. dazu Müller-Graff: Institutionelle und materielle Reformen in der Zusammenarbeit in den Bereichen Justiz und Inneres, S. 259-278; Müller-Graff: Die fortentwickelnde Übernahme des Acquis der „Dritten Säule“ in die „Erste Säule“ der Union, S. 53-75.

53 Vgl. Rebhahn: Art.136 EGV Rn. 4.

54 Vgl. Gegensätze im hohen Norden, S. 12; Häde: Zur Rechtmäßigkeit der Entscheidung über die Europäische Wirtschafts- und Währungsunion, S. 1088-1092.

55 Vgl. dazu u.a.: Umfassender Monitoring-Bericht der Europäischen Kommission über den Stand der Beitrittsvorbereitungen der Tschechischen Republik, Estlands, Zyperns, Lettlands, Litauens, Ungarns, Maltas, Polens, Sloweniens und der Slowakei, $\operatorname{KOM}(2003) 675$ endgültig. 
als Vertragsverletzungsverfahren ohne EuGH bezeichnet werden, weil sie einen schnellen Abwehreingriff der Kommission gegenüber einem Beitrittsstaat ermöglichen. ${ }^{56}$

Derzeit ist die Annahme nicht unrealistisch, dass eine Erweiterung um die Türkei zu einer langzeitigen, wenn nicht dauerhaften Minderintegration dieses Staates führen würde. Anders gesagt: Es käme zu einer ,differenzierten Integration“ der ,Acquis-Staaten “ auf dem Status quo schon allein in Folge eines Beitrittsvertrages. Inwieweit dieser Acquis langfristig doch eine rechtlich und faktisch unitarisierende Einbeziehung in den gesamten Gemeinschaftsstandard nach sich zöge, ist spekulativ.

\section{Das Paradox des Verzichts auf Differenzierung als Sprengsatz für Homogenität}

Die Einbeziehung der Erweiterungsproblematik in die Frage der differenzierten Integration führt beim ersten Anschein auf ein Paradox. Der Verzicht auf Konzepte , differenzierter Integration ' zugunsten des Homogenitätsprinzips ist potenziell ein Sprengsatz für die homogene Integration. Denn ein nicht verwirklichbarer oder nicht gewünschter unionaler Homogenitätsstatus eines Staates oder einzelner Staaten wird Ausweichstrategien derjenigen Staaten hervorrufen, die ein engeres Verhältnis miteinander pflegen wollen: innerhalb oder außerhalb der Union.

Erweiterungen stärken aber innere Spannungen, wenn dadurch der Kern aller Politik, die Bewältigung der als gemeinsam angesehenen Überlebensherausforderungen einer Gruppe, speziell für die Union mit kollidierenden Lösungsansätzen aufgeladen wird, wie sie etwa im Umkreis der Irak-Krise in der Haltung zum ,transatlantischen Führungstopos ‘ sichtbar wurde. Auch dies bedeutet aber, dass sich Integrationsforschung künftig stärker mit den einzelnen Mitgliedstaaten zu befassen hat und nicht nur isoliert mit abstrakten Modellerörterungen für die Union.

\section{Zusammenfassung}

Damit ergibt sich zusammenfassend, dass die konzeptionelle Figur der, differenzierten Integration" innerhalb und außerhalb des einheitlichen institutionellen Rahmens der mitgliederstarken Union als ein Integrationselement mit verschiedenen Funktionen verstanden werden kann: als definierter und kontrollierter Puffer gegen die Erosion des essenziell homogenen Sockels der Rechtsgemeinschaft, als Chance für die Willigen zur Überwindung von Blockaden und als Topos zur Förderung des Zusammenhalts in der Ungewissheit über das Potenzial seiner Sprengkraft. Das Bewusstsein um diese polyfunktionalen Möglichkeiten des Konzepts , differenzierter Integration“ und deren kluge Nutzung kann der konstruktiven Zukunft der Europäischen Union dienlich sein.

56 Referat auf Sachstandtagung des Europäischen Graduiertenkollegs, Systemtransformation und Wirtschaftsintegration im zusammenwachsenden Europa' am 28.10.2005. 